\title{
Network Pharmacology Analysis: A Promising Approach for the Research of Traditional Chinese Medicine
}

\author{
Wenrui Xie ${ }^{1,2}$, Xiaoya Yang ${ }^{3}$, Lihao $\mathrm{Wu}^{1,2}$ and Xingxiang $\mathrm{He}^{1,2^{*}}$ \\ ${ }^{1}$ Department of Gastroenterology, The First Affiliated Hospital of Guangdong Pharmaceutical University, Guangzhou, China; \\ ${ }^{2}$ Research Center for Engineering Techniques of Microbiota-Targeted Therapies of Guangdong Province, The First Affiliated Hospital \\ of Guangdong Pharmaceutical University, Guangzhou, China; ${ }^{3}$ Department of Physiology, Guangzhou Health
}

Science College, Guangzhou, China

\begin{abstract}
Network pharmacology is a new strategy to explore the molecular mechanisms underlying the action of traditional Chinese medicine (TCM) formula by analyzing the biological network of multibiological processes and signaling pathways, and identifying the key components of druggable targets. ${ }^{1,2}$ Network pharmacology approaches have been applied in elucidation of the potential therapeutic effects and action of TCM in several chronic diseases, such as chronic kidney disease, diabetes, rheumatoid arthritis and cancer. ${ }^{1,3}$ Using network pharmacology analysis, Peng ${ }^{4}$ predicted that Xia Sang Ju (XSJ) granule, a traditional TCM, might exert its antihypertensive effects by targeting 11 genes, among which ESR2 and SLC6A2 were the uppermost hypertension-related targets. The author further revealed that XSJ might affect multi-biological processes and multi-pathways to prompt their anti-hypertensive effects, which coincided with the TCM formula's therapy concept of multiple compounds, targets, and pathways. ${ }^{4}$
\end{abstract}

The findings open new avenues for the application of network pharmacology in the study of TCM formula. With the concept of integrity, comprehensiveness and systematic approach, network pharmacology provides new strategies and approaches for the study of TCM formula, because it may change TCM from experience-based medicine to evidence-based medicine. ${ }^{2}$ However, we have to understand that current network pharmacology has limitations. First, the results of network pharmacology analysis are affected by the accuracy and integrity of the related databases. In addition, the efficacy of a TCM formula is dependent on the levels of absorbed constituents in vivo. ${ }^{5,6}$ Thus, it would be more credible and complete if the compounds of XSJ selected in the study were screened from the serum samples for pharmacological experiments. Second, the data from network pharmacology analysis are predictive and static. ${ }^{2}$ Although the study validated by a virtual strategy, the results still need further experimental validation in vivo or in vitro to exclude potential false positives and observe the dynamic process of XSJ for antihypertensive actions. Third, the research on TCM formula employed network pharmacology and mainly provided qualitative analysis results, and there was little reported on the quantity of

Abbreviations: TCM, traditional Chinese medicine; XSJ, Xia Sang Ju.

Received: August 13, 2020; Revised: August 14, 2020; Accepted: August 19, 2020

*Correspondence to: Xingxiang He, Department of Gastroenterology, The First Affiliated Hospital of Guangdong Pharmaceutical University, 19 Nonglinxia Road, Guangzhou 510080, China. Tel: +86-020-6132-1457, Fax: +86-020-6132-1457, Email: hexingxiang@gdpu.edu.cn

How to cite this article: Xie W, Yang X, Wu L, He X. Network Pharmacology Analysis: A Promising Approach for the Research of Traditional Chinese Medicine. $J$ Explor Res Pharmacol 2020;5(4):49-50. doi: 10.14218/JERP.2020.00024. these constituents. ${ }^{2}$ In the future, how to achieve a quantitative analysis will be a new challenge for the development of network pharmacology.

Acknowledgments

None.

Funding

This paper was supported by the Natural Science Foundation of Guangdong Province, China (No. 2019A1515010125, XXH) and Department of Education of Guangdong Province (No. 2020KZDZX1132, XXH; No. 2018GKTSCX033, XYY).

Conflict of interest

The authors declare that they have no competing interests.

Author contributions

Manuscript writing (WRX, XYY), and supports of administration or intellectual content (LHW, XXH).

\section{References}

[1] Hao DC, Xiao PG. Network pharmacology: a Rosetta Stone for traditional Chinese medicine. Drug Dev Res 2014;75(5):299-312. doi:10.1002/ddr.21214

[2] Luo TT, Lu Y, Yan SK, Xiao X, Rong XL, Guo J. Network Pharmacology in Research of Chinese Medicine Formula: Methodology, Application and Prospective. Chin J Integr Med 2020;26(1):72-80. doi:10.1007/ s11655-019-3064-0.

[3] Yuan H, Ma Q, Cui H, Liu G, Zhao X, Li W, et al. How Can Synergism of Traditional Medicines Benefit from Network Pharmacology? Molecules 2017;22(7):1135. doi:10.3390/molecules22071135.

[4] Peng M. Network Pharmacology Analysis Uncovers the Potential Anti-Hypertensive Mechanisms of Xia Sang Ju Granule. J Explor Res Pharmacol 2020;doi:10.14218/jerp.2020.00008.

[5] Zhang X, Pi Z, Zheng Z, Liu Z, Song F. Comprehensive investigation of in-vivo ingredients and action mechanism of iridoid extract from Gardeniae Fructus by liquid chromatography combined with mass 
J Explor Res Pharmacol

spectrometry, microdialysis sampling and network pharmacology. J Chromatogr B Analyt Technol Biomed Life Sci 2018;1076:70-76. doi:10.1016/j.jchromb.2018.01.023.

[6] Yu S, Liu H, Li K, Qin Z, Qin X, Zhu P, et al. Rapid characterization
Xie W. et al: Network pharmacology analysis for TCM

of the absorbed constituents in rat serum after oral administration and action mechanism of Naozhenning granule using LC-MS and network pharmacology. J Pharm Biomed Anal 2019;166:281-290. doi:10.1016/j.jpba.2019.01.020. 